\title{
Cost considerations in the management of atrial fibrillation - impact of dronedarone
}

This article was published in the following Dove Press journal:

ClinicoEconomics and Outcomes Research

5 March 2012

Number of times this article has been viewed

\author{
Yaariv Khaykin ${ }^{1,2}$ \\ Yana Shamiss' \\ 'Heart Rhythm Program, Southlake \\ Regional Health Center, Newmarket, \\ Ontario, Canada; ${ }^{2}$ Faculty of Medicine, \\ University of Toronto, Ontario, \\ Canada
}

\begin{abstract}
Atrial fibrillation (AF) is the most common sustained cardiac arrhythmia. It is associated with significant morbidity and mortality. At the societal level, AF carries an enormous cost. Strategies aimed at reducing AF morbidity and mortality and containing the associated fiscal burden are of paramount importance. This review will discuss AF treatment strategies and economics, focusing on the impact of dronedarone, a novel antiarrhythmic agent.
\end{abstract}

Keywords: antiarrhythmic, rhythm control, heart failure, ablation, costs

\section{Introduction}

Atrial Fibrillation (AF) is the most common sustained cardiac arrhythmia affecting three million Americans with prevalence expected to reach ten million by $2050 .^{1-3}$ Its occurrence rises with age with as many as $10 \%$ of the population over 80 years of age afflicted. It is responsible for most arrhythmia-related hospitalizations and leads to the greatest length of hospital stay associated with any disorder of the cardiac rhythm. ${ }^{4}$

While asymptomatic in some patients, $\mathrm{AF}$ is a source of significant disability in others. It may present with palpitations in younger patients with preserved diastolic function, less dependent on atrial contraction, and with symptoms of congestive heart failure in patients with hypertension or cardiomyopathy, where controlled heart rate and atrial "kick" are of paramount importance to ventricular filling. ${ }^{5} \mathrm{AF}$ is responsible for up to $30 \%$ of all ischemic strokes, a source of significant disability and mortality in these patients. ${ }^{6}$ The risk of stroke is higher in AF patients over 75 as well as in patients with history of hypertension, diabetes, congestive heart failure, and prior embolic events, all commonly present in this group. ${ }^{7}$ Among patients enrolled in the Framingham study, mortality in AF patients was higher by a factor of 1.5 among men and 1.9 among women. ${ }^{8}$

Apart from its clinical impact, $\mathrm{AF}$ carries an enormous fiscal burden. This relates to the cost of physician visits, hospital admissions, tests and invasive procedures, medications and over-the-counter alternatives, as well as the cost related to the treatment of comorbidities and complications. Several recent studies have gone beyond addressing these issues and reported on the lost productivity related to AF, ranging from 9 to 26 days of work per year with a significant increase in short-term disability. ${ }^{9}$

Strategies aimed at reducing AF related complications and costs are critical and will be discussed in this review with focus on the impact of dronedarone. 


\section{Current strategies in the management of AF}

Management strategies for AF fall into one of three main categories - symptom relief and management of congestive heart failure, prevention of thromboembolic complications, and rate control. All of these deserve to be addressed in each individual patient, while strategies used to address one of these areas may also help to impact others.

Symptom relief most commonly comes in the form of rate control in patients with persistent or permanent forms of AF, while patients with the paroxysmal form of this condition may benefit from rhythm control in order to improve their quality of life. There is little evidence that one strategy is superior to the other in terms of morbidity or mortality, however, it is clear that patients who are in fact able to stay in rhythm do better over time. ${ }^{10-12}$

Unfortunately, rhythm control in AF patients can only be achieved with antiarrhythmic medications $40 \%-60 \%$ of the time because of their limited efficacy and significant associated side effects. ${ }^{13}$ Amiodarone, the most effective antiarrhythmic medication on the market, is also the most toxic, negatively affecting a variety of organ systems. Its efficacy comes in part from an extremely long half-life, which may allow the patient to miss several doses of amiodarone without any noticeable clinical impact. At the same time, toxicities related to amiodarone are cumulative and the likelihood of adverse events goes up with the duration of exposure and total dose administered over time. ${ }^{14}$ Other antiarrhythmic medications may be outright dangerous in certain populations. Sotalol and dofetilide may lead to QT interval prolongation and ventricular fibrillation in some patients and cannot be administered to patients with renal dysfunction. Sotalol is poorly tolerated by patients with congestive heart failure and may result in disabling fatigue in others. Class I agents such as flecainide and propafenone may cause ventricular tachyarrhythmia in patients with structural heart disease and, particularly, those with history of ischemic cardiomyopathy. These drugs can also convert AF to atrial flutter and paradoxically, by lowering the atrial rate, facilitate 1:1 atrioventricular (AV) nodal conduction. Safe administration of Class I drugs involves co-administration of AV nodal blocking agents. Unfortunately, sotalol, and other AV nodal blocking agents which may be used for rate control, or co-administered with Class I agents, can exacerbate sinus node dysfunction, highly prevalent among patients with $\mathrm{AF}$, and potentially lead to the need for permanent pacemaker placement. ${ }^{15}$

Ultimately, catheter ablation for AF has developed over the last decade to help alleviate symptoms. While superior to antiarrhythmic drugs this approach does not appear to offer a cure to a number of patients, but rather appears to delay progression of the disease and does carry with it up to $4.3 \%$ risk of significant complications based on a recently published worldwide survey of the ablating centers. ${ }^{16,17}$

Rate-control strategy, while important to consider in symptom management, has an independent value as well. Some AF patients react poorly to the rapid AV nodal conduction and develop so called "tachycardia-mediated" cardiomyopathy. These patients may suffer from congestive heart failure or may present without symptoms but with clear deterioration in their left ventricular ejection fraction. Fortunately, at least in some patients changes reverse with adequate rate control. The concept of what rate qualifies as adequate has seen some changes recently as the investigators of the RACE II trial reported little difference between aggressive rate control targeting a resting rate of 80 beats per minute (bpm) and the more lenient approach aiming at $110 \mathrm{bpm} .{ }^{18}$ Most patients in either arm of the study ended up with a ventricular rate below $100 \mathrm{bpm}$, a cutoff incorporated into the recently revised Canadian Cardiovascular Society guidelines on AF management. ${ }^{19}$ Rate control is typically achieved with AV nodal blocking agents such as beta blockers and non-dihydropyridine calcium channel blockers, but there is still room for the less effective digitalis, particularly among heart failure patients, those with diminished left ventricular ejection fraction, or as a second or third line agent.

Prevention of embolic complications is the most important aspect of care for AF patients. These range from transient ischemic events (TIA) to strokes and are the most costly complication of AF. Strokes secondary to AF are more severe than those secondary to atherosclerotic disease and impart a greater disability on the victims..$^{20}$ This results in significant costs related to hospitalizations, rehabilitation, and chronic disability. Strategies aimed at reducing embolic events in AF patients include therapy with aspirin, combination of aspirin and clopidogrel, and oral anticoagulant therapy with warfarin or one of the new agents targeting either thrombin or Factor $\mathrm{Xa}{ }^{21-23}$ While effective from the point of view of preventing strokes and other embolic events, one must be aware of the significant risk of bleeding associated with these agents used alone, and especially, in combination.

One other strategy aiming to minimize the risk of embolic events involves mechanical elimination or closure of the left atrial appendage, the area where clots related to AF most commonly form. Techniques for left atrial appendage closure or excision have been initially developed by the cardiac surgeons. ${ }^{24}$ Novel left atrial appendage closure devices 
have recently shown promise in reducing the risk of stroke in patients who cannot take antithrombotic agents and can be placed percutaneously. ${ }^{25}$

\section{Cost of AF and its management}

A number of studies have looked at the costs related to AF across the world. One must be cautious when evaluating their findings since the data comes from a potpourri of sources ranging from administrative databases, to multicenter and national registries, to single center studies. Patient populations described are also heterogeneous and range from the relatively young and healthy but highly symptomatic patients presenting for AF ablation, to older patients admitted to the hospitals with embolic sequelae of AF.

Multiple negative health outcomes in AF patients as well as AF treatment strategies contribute to an ever-growing tap drawn on the healthcare system and the society at large. A recent systematic review of the cost of AF care revealed that the overall average annual cost to manage one AF patient is US\$7015 in 2010 with a range of estimated costs as high as just over US $\$ 10,000 .{ }^{26}$ While these costs are substantial, they represent only about one quarter of the entire health system costs for patients with AF. A German study estimated the entire annual cost of care for patients with a stroke secondary to $\mathrm{AF}$ at $\$ 20,613 .{ }^{27}$ While an American study estimated annual cost of care for an AF patient at \$40,169. ${ }^{6}$ Hospitalizations are the most important determinant of the total cost (58\%) with the cost of a single acute admission in Ontario with AF as a primary diagnosis of US\$23,392 in $2010 .{ }^{28}$ Similarly, direct costs attributable to AF in the US, based on the findings from an insurance database, were $\$ 15,553$ per year in 2002 with $75 \%$ of the cost related to in-patient care. ${ }^{26,29}$ Each AF related hospitalization in another group of Medicare insured patients cost an average of US\$11,085 (2004-2007), further supporting these findings. ${ }^{30}$ To make matters worse, $50 \%$ of the AF patients may be readmitted within a year, leading to further rising costs.

A number of AF cost estimates have been published internationally. In a recent analysis of a German insurance database, close to $80 \%$ of the cost of care in the first year following an AF related hospitalization was due to the index event with $15 \%$ attributable to the cost of drugs, and $3 \%$ to the outpatient care. ${ }^{31}$ The cost of non-traditional adjuvants and remedies as well as that of sickness benefits, typically not included in other AF cost analyses, was on par with the cost of outpatient care - contributing about $4 \%$ of the overall treatment cost, which came in at an astounding 7,688 \pm 954 Euro per patient in 2005 currency.
It does not come as a surprise that the cost reported using administrative databases is substantially higher than the estimates from surveys and "back of the envelope" exercises reported for a number of geographies. Euro Heart Survey on AF published estimated annual costs of AF care ranging from $€ 698$ in Poland to $€ 1544$ in the Netherlands in $2006^{32}$ or an annual cost of care in an AF patient of US\$4840 in 2005 previously estimated by our group. ${ }^{33}$ Treatment costs associated with follow-up of AF patients including hospital admissions, emergency room visits, testing, and follow-up with cardiologists, internists, and family physicians were also reported in France. ${ }^{34}$ This analysis stratified patients according to therapeutic strategy - rate or rhythm control as well as according to concomitant congestive heart failure symptoms. The authors estimated the average total 5-year cost of AF at $€ 16,539$ in 1998 currency.

The real cost of care for an AF patient likely lies somewhere in between with surveys underestimating some of the less apparent cost contributors and counting AF as the primary diagnosis responsible for treatment and associated costs, and administrative databases overestimating costs related to AF as a secondary diagnosis in typically older and sicker cohorts of insured patients than those studied in the surveys.

As a result, system cost attributable to AF is staggering with over US\$2 billion spent only on the care of patients with AF-related strokes in the US Medicare system and a total estimated medical expenditure related to AF around US\$6.5 billion per year. ${ }^{26}$

\section{Cost containment strategies}

A number of studies have looked at the potential cost containment strategies. Of these the most obvious is greater attention to anticoagulation therapy in these patients. The bulk of the current cost of AF care is related to thromboembolism, yet currently as few as $10 \%-20 \%$ of the AF patients are treated with appropriate prophylaxis strategies. ${ }^{35}$ Those who do take oral anticoagulants spend much of their time taking subtherapeutic doses of the medication placing them at risk of stroke, while others take supertherapeutic doses and run a significant risk of bleeding given a very narrow therapeutic range of these drugs. Novel antithrombotic agents allow for more consistent anticoagulation and have been shown superior to warfarin in stroke prevention.

In the study of Dagibatran versus Warfarin in Patients with Atrial Fibrillation (RELY) the use of dabigatran, a direct thrombin inhibitor, was associated with similar rates of stroke and systemic embolism but lower rate of major bleeding 
compared to warfarin at a lower dose of $110 \mathrm{mg}$, while the higher dose of the drug at $150 \mathrm{mg}$ was associated with lower rates of stroke and systemic embolism but similar rates of major bleeding compared to warfarin. ${ }^{23}$ Similarly, in the study of Apixaban versus Warfarin in Patients with Atrial Fibrillation (ARISTOTLE), apixaban, a factor Xa inhibitor, was superior to warfarin in preventing stroke or systemic embolism, caused less bleeding, and resulted in lower mortality. ${ }^{36}$ Based on their better safety profile these medications will likely result in further savings compared to warfarin. Indeed, when analysed within the Canadian healthcare system, the incremental cost effectiveness ratio (ICER) of dabigatran was \$10,440/ qualityadjusted life-years (QALY) versus warfarin and \$3962/ QALY versus "real-world" prescribing. ${ }^{37}$ The estimates were more conservative within the UK healthcare system at $£ 23,082-$ $£ 42,386 /$ QALY. $^{38}$ A similar US analysis found it to be less so at an ICER of $\$ 86,000 /$ QALY. ${ }^{39}$ The differences between these are explained by different stratification of the patients by CHADS-2 score and assumptions with respect to INR control and are beyond the scope of this paper.

Another such strategy has to do with ablation. First promise for a potential cure for AF came in 1998 when it became apparent that ectopic atrial activity originating in the pulmonary veins may be responsible for initiation of AF and could be targeted with radiofrequency energy. ${ }^{40}$ The field of targeting AF triggers has seen substantial progress since this discovery with multiple tools coming to market over the last decade in an effort to improve the safety and efficacy of these procedures. Most of these strategies involve delivering various types of energy just proximal to the insertion of the pulmonary veins into the left atrium using conventional, irrigated tip, circular, and balloon shaped catheters. Another strategy that was first described in 2004 and has seen much technological attention has been that of targeting tissues thought to perpetuate AF or presenting so called AF substrate. ${ }^{41}$ These latter efforts have focused on elimination of the viable atrial myocardium displaying particularly disorganized activity during AF, or delivering energy over autonomic nerve ganglia thought to initiate and perpetuate the arrhythmia. ${ }^{42}$

These approaches have shown promise in a multitude of individual center and multicenter randomized trials uniformly showing clinical benefit of ablation over antiarrhythmic drug therapy with respect to sinus rhythm maintenance, quality of life, and arrhythmia related hospitalizations in at least some populations. ${ }^{16,43,44}$

Several projections of cost of care of an AF patient have been published in an attempt to estimate the relative cost of ablation and contrast it to the cost of medical therapy over time. A study directly comparing the costs of ablation and medical therapy in the Canadian healthcare environment has been published. ${ }^{33}$ Costs related to medical therapy in the analysis included the cost of anticoagulation, rate and rhythm control medications, non-invasive testing, physician follow-up visits, and hospital admissions, as well as the cost of complications related to this management strategy. Costs related to catheter ablation were assumed to include the cost of the ablation tools (electroanatomic mapping or intracardiac echocardiography-guided pulmonary vein ablation), hospital and physician billings, costs related to periprocedural medical care and complications. Costs related to these various elements were obtained from the Canadian Registry of Atrial Fibrillation (CARAF), government fee schedules, and published data. Sensitivity analyses looking at a range of initial success rates $(50 \%-75 \%)$ and late attrition rates ( $1 \%-5 \%)$, prevalence of congestive heart failure $(20 \%-60 \%)$, as well as discounting varying from $3 \%$ to $5 \%$ per year were performed. In this study, the cost of catheter ablation strategy ranged from $\sim$ US $\$ 14,000$ to US\$18,000 in 2005 currency. It was assumed that patients who required anticoagulation prior to ablation would continue on this therapy following the procedure with an annual average follow-up cost of US\$1400 to US\$1800 among the ablated patients. The annual cost of medical therapy ranged from US\$3600 to US\$4300. The latter estimate was supported by the findings from the FRACTAL registry which prospectively collected clinical and cost data for 973 patients with $\mathrm{AF}^{45}$ The study projected costs of ongoing medical therapy and catheter ablation to equalize at 3.2 to 8.4 years of follow-up in this study but did not take into account development of the novel antiarrhythmic and thromboprophylactic strategies not available at the time of the publication.

Four papers attempted to perform a cost-benefit analysis of AF ablation with that of medical therapy. In the first of these studies, a Markov decision analysis model looking at 55- and 65-year-old cohorts of patients at low and moderate risk of stroke was created by the investigators. ${ }^{46}$ Complications and costs related to AF, medical therapy, and catheter ablation were accounted for. The model assumed that amiodarone would be used for rhythm control and a combination of digoxin and atenolol for rate control. Eighty percent efficacy of AF ablation was assumed with $30 \%$ redo rate during the first year and $2 \%$ per year late success attrition rate. It was further assumed that as many as $38 \%$ of the patients on rate control would convert to sinus rhythm with annual AF relapse rate of 5\%. Moderate risk of stroke 
was defined as having one risk factor, including diabetes, hypertension, coronary artery disease, or congestive heart failure. Patients at low risk of stroke were assumed to have no such risk factors. For the purpose of the model, patients at moderate risk of stroke were anticoagulated whereas those at low risk could be on warfarin or aspirin. The model incorporated an annual stroke risk of $2.3 \%$ and $1.1 \%$ for patients treated with aspirin and $1.3 \%$ and $0.7 \%$ for those on warfarin at moderate and low risk for stroke respectively. A relative stroke risk of $1.4 \%$ per decade was accounted for. Age adjusted mortality based on life tables and mortality reductions attributable to aspirin and warfarin were accounted for. All healthcare costs were calculated in 2004 US dollars using 3\% discounting per year. Costs were estimated based on Medicare reimbursement rates, hospital accounting information, published literature, and the Red Book for wholesale drug costs. Catheter ablation appeared to be most cost-effective in younger patients at moderate risk of stroke at $\$ 28,700 / \mathrm{QALY}$ gained. It was somewhat less cost effective in the older moderate risk patients at $\$ 51,800 / \mathrm{QALY}$ gained and least cost-effective among the younger patients at low risk of stroke at $\$ 98,900 /$ QALY gained. Unfortunately, since no evidence has been presented to date on the efficacy of ablation for prevention of thromboembolic events, the findings of this study are conditional on such evidence coming to light in the years to come.

Eckard et al developed a decision-analytic model to estimate costs, health outcomes, and incremental costeffectiveness of RFA compared to AAD treatment for AF for a lifetime time horizon. ${ }^{47}$ The authors used a decision tree for the initial year in which the RFA procedure was assumed to take place, and a long-term Markov structure for subsequent years. The authors factored in the potential for a second ablation within a year of the first procedure in patients still suffering from AF. They assumed $70 \%-80 \%$ ablation success within the first year with 1.4 ablations per patient required to maintain rhythm based on Swedish data. The cost of ablation was estimated at around US\$12,000 in 2006, including the cost of 3-4 days in hospital, all diagnostic examinations necessary as well as the cost of disposables. Annual cost of AF therapy was estimated at US\$2000. In order to estimate QALY weights for different health states, age-adjusted QALY weights based on a Swedish general population were applied for patients in the controlled AF state, and used as reference points. A decrement of 0.1 for uncontrolled AF and 0.25 for stroke was applied to the baseline utility in the controlled AF state. With annual success attrition rates of $5 \%, 10 \%$, and $15 \%$ used in the sensitivity analysis, the relative cost of ablation was estimated up to US\$58,000 per QALY without assuming stroke prevention related to the ablation strategy.

A similar analysis in the United Kingdom suggested incremental cost effectiveness of ablation at US $\$ 16,000$ per QALY in 2008. The authors of this paper assumed freedom from $\mathrm{AF}$ at $84 \%$ at one year with $2 \%-4 \% /$ year rate of success attrition over time resulting in their estimates favoring ablation over the other published economic analyses. Further sensitivity analyses found the estimate to depend significantly both on the relative quality of life estimate associated with sinus rhythm and on the prognostic implications of being in rhythm. ${ }^{48}$

Finally in a more recent paper, Reynolds and his group published a Markov model cost effectiveness analysis of ablation versus antiarrythmic therapy in a simulated cohort of patients with paroxysmal drug refractory AF projected over 5 years. The authors assumed $60 \%$ success of the ablation approach with a $25 \%$ rate of repeat ablation. Utilities for quality of life assessment were derived from real-life data, using the FRACTAL registry for the medically treated patients using SF-12 and patients ablated at the authors' institution, as well as those enrolled in the A4 trial for derivation of the scores in this cohort based on the SF-36 questionnaire. In the base scenario, the incremental cost per QALY among ablated patients was US $\$ 47,333$ in 2009 with cost neutrality achieved at $\sim 10$ years taking into account $3 \%$ discounting. ${ }^{49}$

At the same time, given that the most expensive aspect of AF care relates to hospitalizations, strategies which may decrease the need for, and the length of, hospitalizations would be expected to lead to a significant reduction in the economic burden of AF.

\section{Impact of dronedarone}

Dronedarone is a novel antiarrhythmic agent developed on the basis of the amiodarone molecule. ${ }^{50}$ Pharmacologically, the molecule of dronedarone does not carry iodine, thought to account for most of the end-organ toxicity seen among amiodarone-treated patients. It was also modified to make it more hydrophilic and to expedite elimination half-life compared to its parent drug. Dronedarone had undergone extensive clinical testing in multiple trials and was shown to have rhythm control efficacy comparable to that of sotalol or class Ic agents (Table 1). In addition dronedarone has been shown to provide a measure of rate control, lowering heart rate in AF among treated patients by an average of $14 \mathrm{bpm} .{ }^{51}$ Unlike other antiarrhythmic agents, dronedarone may be started on an outpatient basis without the need for in-patient 


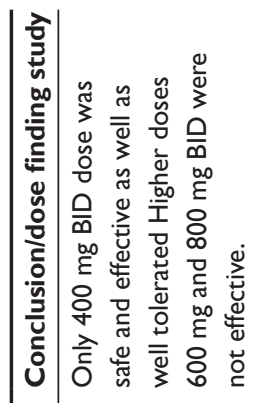

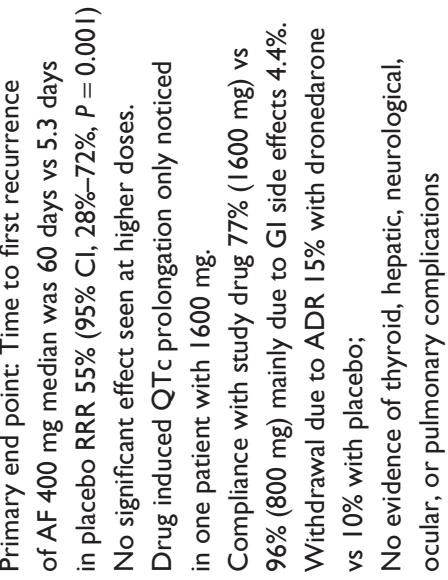

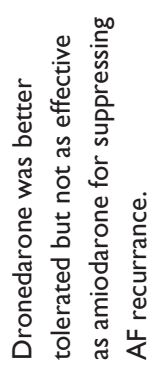

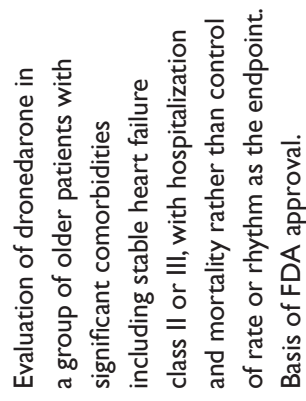

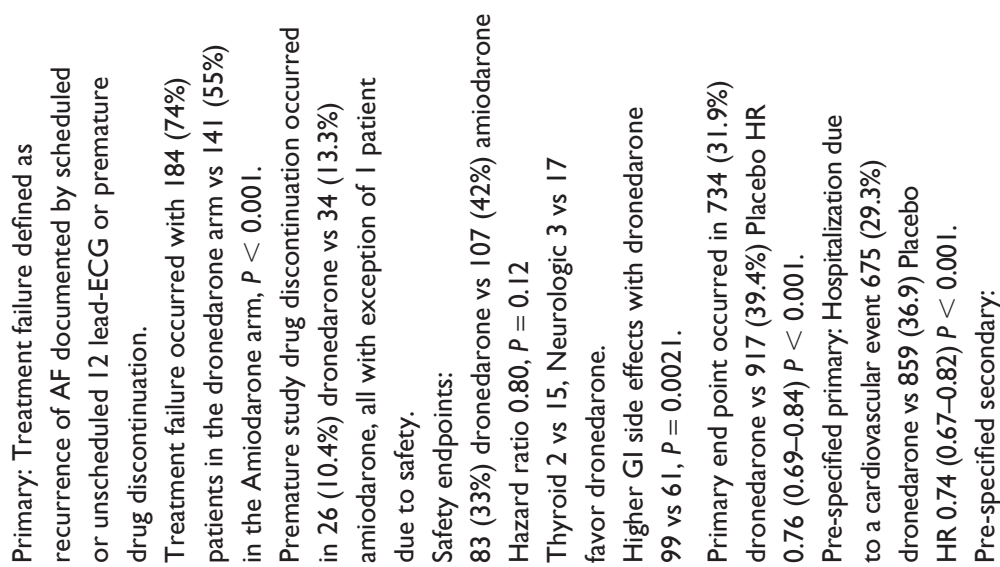

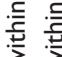

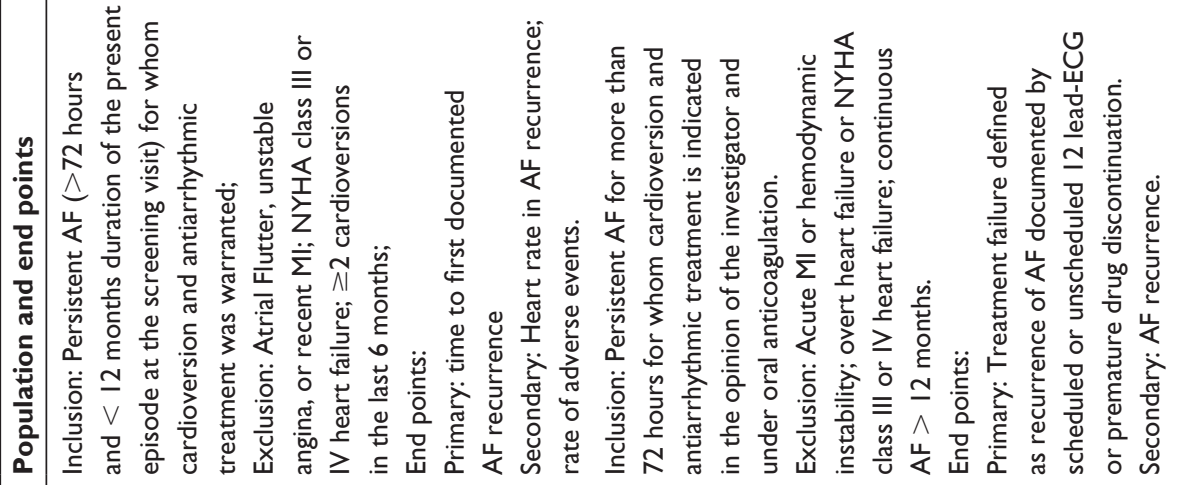

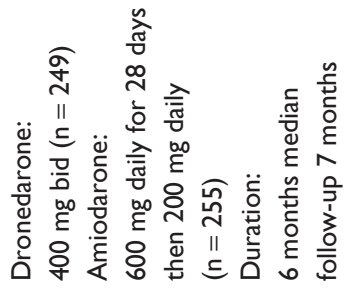
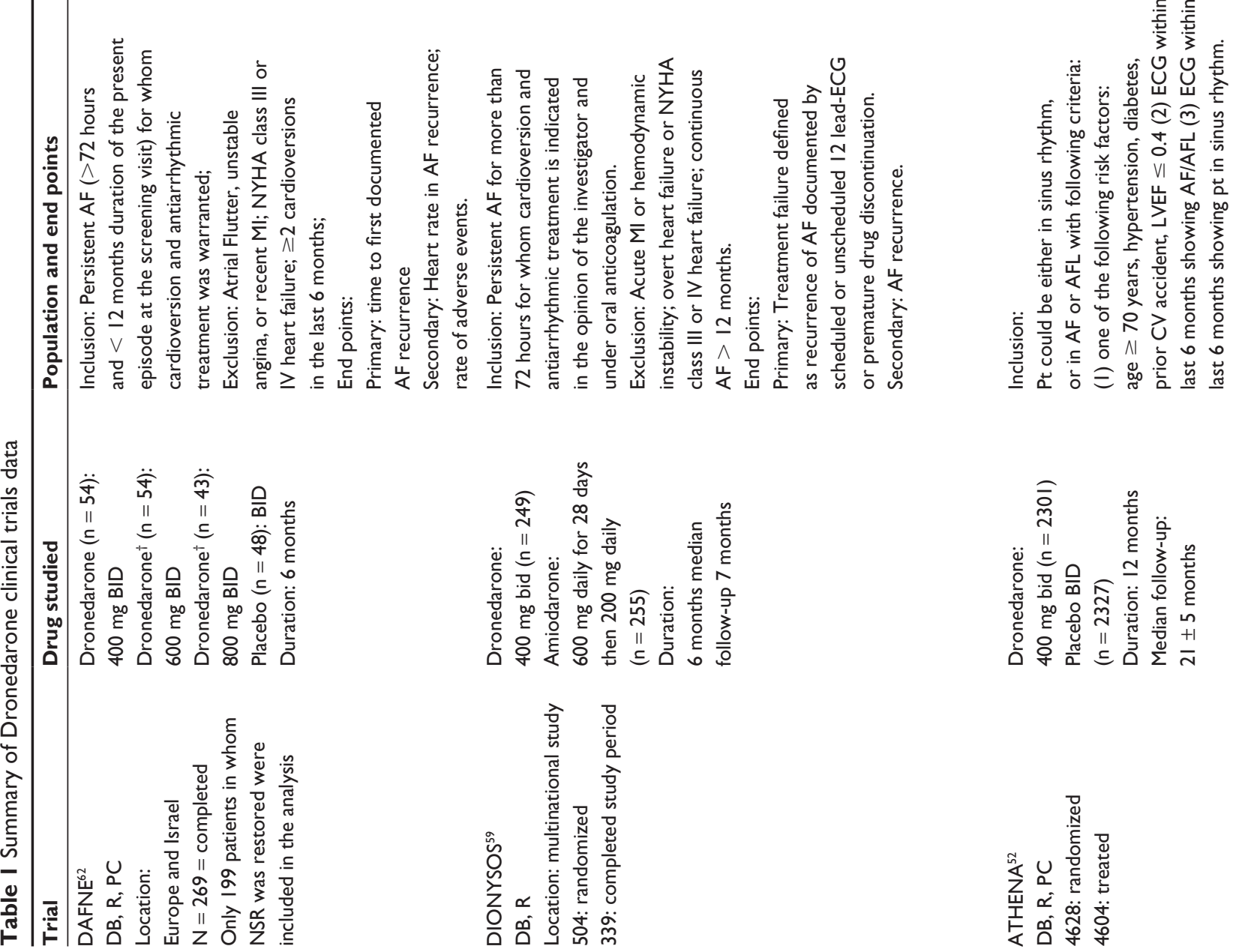

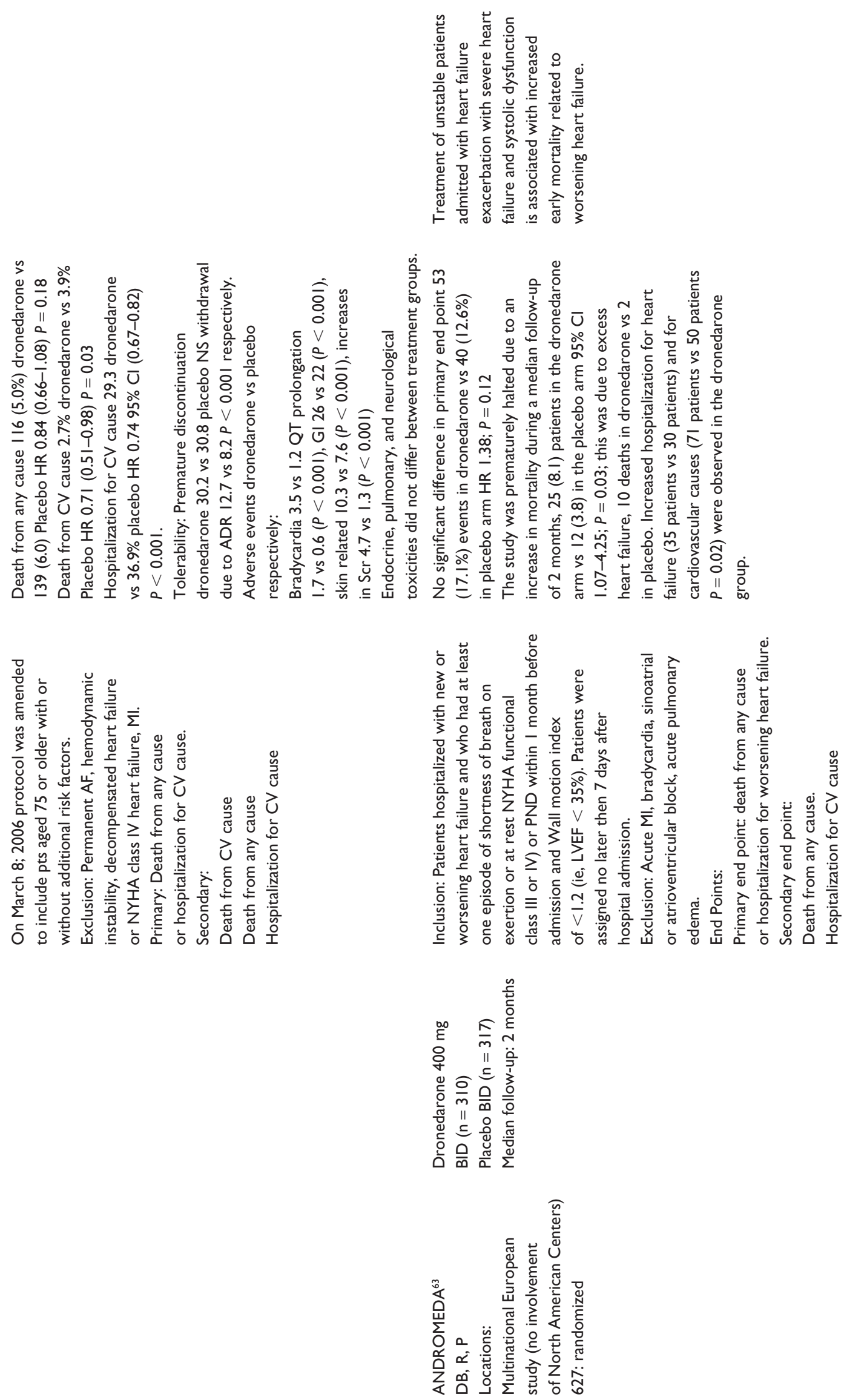

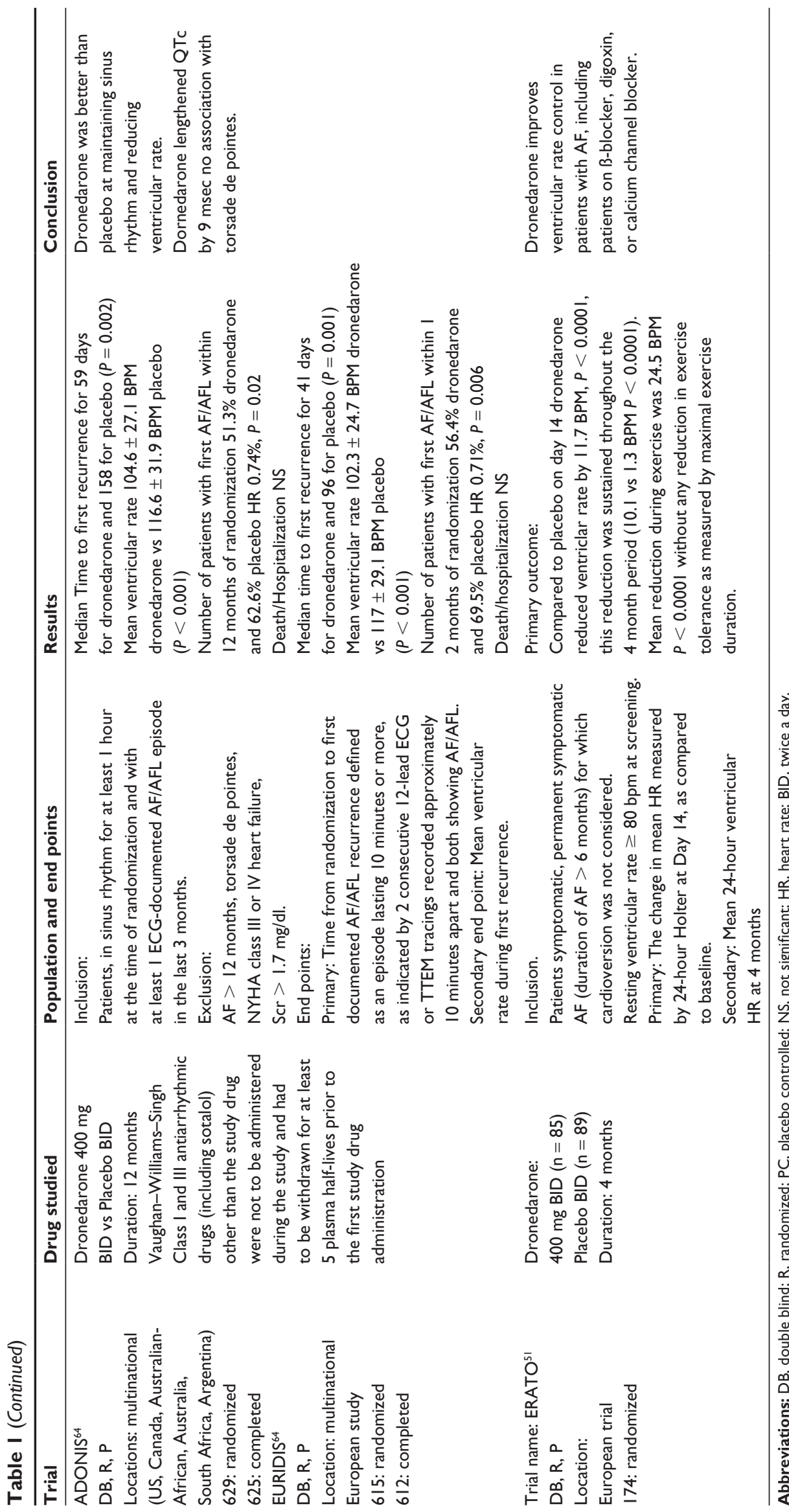
monitoring required for sotalol, propafenone, flecainide, and dofetilide.

ATHENA, a double blind placebo controlled trial studied the effects of dronedarone in addition to standard therapy in patients with risk factors including age over 75 , or age under 75 with at least one of hypertension, diabetes, stroke or TIA, enlarged left atrial dimension $(>50 \mathrm{~mm})$, or reduced left ventricular ejection fraction $(<40 \%) .{ }^{52}$ Dronedarone was shown to reduce AF related hospitalizations with a hazard ratio of 0.626 compared to placebo, ${ }^{53}$ in a similar population to that reported by Reinhold and colleagues. ${ }^{31}$ Dronedarone also reduced duration of hospitalization and the risk of stroke in these patients by close to $40 \%$. Both of these effects would potentially reduce the cost of care by $€ 2875$ per patient per year based on the German data or approximately US $\$ 3000$ 6000 based on the US and Canadian data.

In a study of the Humana registry of patients and physicians, the authors administered an AF continuing medical education (CME) activity, which included information on ATHENA, to the participating healthcare providers (unpublished data). They then independently tested whether the physicians internalized the information provided in the CME and looked at the costs of AF management before and after CME was administered. Study participants demonstrated a significant gain in knowledge and confidence related to the care of AF patients following CME. The authors were able to show that the use of dronedarone increased 2.5-fold during the study period, whereas little changed with respect to the use of oral anticoagulants in the study with only $49 \%$ of the guidelineeligible patients on this therapy. Nevertheless, there was a significant decline in the number and duration of AF-related hospitalizations and a corresponding decline in AF-related healthcare expenditure.

A cost-effectiveness analysis using a discrete event simulation that predicts a person's course if they are treated with dronedarone compared with the predicted course with alternative treatment pathways was included as part of the regulatory submission to the British National Institute for Health and Clinical Excellence (NICE).$^{54}$ In this analysis patients were stratified depending on their type of AF and baseline risk factors into five groups: paroxysmal AF without structural heart disease, paroxysmal AF with coronary heart disease, paroxysmal AF with left ventricular dysfunction, persistent AF without structural heart disease, and persistent AF with structural heart disease. When dronedarone was evaluated as part of a first-line treatment for people with a CHADS- 2 score of 4 or more (in addition to standard baseline therapy), the comparator was standard baseline therapy alone (including beta blockers and anticoagulation). When dronedarone was evaluated as a second-line treatment option, the comparators were the antiarrhythmic drugs amiodarone, sotalol, and class Ic drugs, depending on the type of AF and baseline risk factors.

The model used a lifetime time horizon and included four health states: normal sinus rhythm, permanent AF with uncontrolled symptoms, permanent AF with controlled symptoms, and death. From the normal sinus rhythm state, people could move to any of the other states. From the two permanent AF health states, people could move between these states or to death. Transition between health states was determined by the following events: AF recurrence, acute coronary syndrome, stroke, congestive heart failure, treatment discontinuation, change in symptoms (for the permanent AF states), or death. The baseline risk of these events was taken from the ATHENA trial, extrapolated to a lifetime time horizon and adjusted for each treatment arm using odds ratios from the mixed treatment comparison. All-cause mortality was estimated using age-specific UK life tables and adjusted for CHADS-2 score. The risk of death after stroke and congestive heart failure events was estimated using published sources. The model also included adverse events associated with each treatment.

Drug costs were derived from the "British national formulary" (edition 57). Drug administration costs for dronedarone consisted of a specialist outpatient visit for treatment initiation and a GP visit for a day-7 creatinine test (£213). For comparators, it was assumed that hospitalization was required for treatment initiation (£249) and 6-monthly GP visits and tests were required for monitoring (£58-£76 depending on the treatment). Costs for the majority of health events occurring in the model were taken from published literature. Most events were assumed to incur a one-off cost; but for stroke and congestive heart failure, ongoing daily costs were assumed. Costs for adverse events came from NHS Reference Costs 2007-2008. A proportion of adverse events were assumed to require hospitalization (based on expert clinical opinion) and the rest were assumed to require an outpatient consultant visit. For short-term adverse events, a one-off cost at treatment initiation was incurred and for adverse events with lifetime effects, a 6-monthly GP visit was assumed to be required. Data on resource use were sourced from clinical opinion and published literature.

In the base-case analysis, the incremental costeffectiveness ratios (ICERs) for the analysis of dronedarone if given in addition to standard baseline therapy (for people with a CHADS- 2 score of 4 or more) compared with standard 
baseline therapy alone ranged from $£ 6757$ to $£ 7890$ per QALY gained (incremental costs $£ 3053$ and $£ 3307$ and incremental benefits 0.45 and 0.42 QALYs for these two ICERs respectively). The ICERs varied depending on the type of AF and the presence of structural heart disease, coronary heart disease, or left ventricular dysfunction. For the analysis of dronedarone as an alternative antiarrhythmic drug to amiodarone, the ICERs were $£ 2645$ per QALY gained (incremental cost $£ 3528$ and incremental benefit 1.33 QALYs) for paroxysmal AF with left ventricular dysfunction and $£ 3113$ per QALY gained (incremental cost $£ 3986$ and incremental benefit 1.28 QALYs) for persistent AF with structural heart disease. For the comparison of dronedarone with class Ic drugs, the ICERs were $£ 20,003$ per QALY gained (incremental cost $£ 1980$ and incremental benefit 0.10 QALYs) for paroxysmal AF with no structural heart disease and $£ 20,761$ per QALY gained (incremental cost $£ 2069$ and incremental benefit 0.10 QALYs) for persistent AF with no structural heart disease. For the comparison of dronedarone with sotalol, the ICERs ranged from $£ 1929$ to $£ 2197$ per QALY gained (incremental costs $£ 3986$ and $£ 4384$ and incremental benefits 2.07 and 2.00 QALYs for these two ICERs respectively) (depending on the type of AF and the presence or absence of underlying heart disease). Further analysis by the Economic Review Group of NICE concluded that the model hinged on the assumption that dronedarone lowers mortality and that regrettably it does not specifically account for a lower rate of hospitalizations related to the drug. ${ }^{54}$

Unfortunately, not all patients with AF benefit from this agent. So in the study of dronedarone administered to patients with a recent heart failure hospitalization, dronedarone was associated with increased mortality and the study was terminated early. ${ }^{55}$ A similar outcome was seen in the cohort of AF patients suffering from the persistent form of this condition who also had history of reduced left ventricular ejection fraction and congestive heart failure. ${ }^{56}$

Dronedarone is not free of interactions - it increases the levels of dabigatran, a novel oral anticoagulant, which may increase the risk of bleeding in this growing group of patients. It has been associated with significant liver toxicity and requires routine follow-up of the liver function studies. ${ }^{57}$ Finally, it is not as effective as amiodarone from the point of view of rhythm control, desired by some patients and physicians. ${ }^{58,59}$

Based on these limitations, the role of dronedarone promising at the time of its entry onto the market is less certain. Given available data it should be used in patients with paroxysmal AF and without history of significant left ventricular dysfunction, or congestive heart failure symptoms who are not at significant risk for liver disease. Patients treated with dronedarone should have their liver profile followed closely while on the drug. While the mindset of most physicians involves the use of dronedarone like any other antiarrhythmic agent, the data to support this practice is scant. On the other hand, dronedarone has excellent data to support its use in patients over 75 and those over 70 with one of the CHADS-2 risk factors for stroke for prognostic reasons, along the lines of beta blockers, statins, and $\mathrm{ACE}$ inhibitors, rather than to achieve rhythm control per se with excellent ICERs if the assumption of lower mortality holds true, or better still if hospitalizations are accounted for.

This subtlety was not appreciated by the The Canadian Expert Drug Advisory Committee (CEDAC), which recommended not listing the drug for coverage. ${ }^{60}$ Their decision was based on the fact that dronedarone was several times more expensive than amiodarone while being inferior as an antiarrhythmic agent. The Committee felt that the evidence for dronedarone being safer than amiodarone was not substantiated well enough despite admitting that the risk of adverse neurological and thyroid events was lower among patients treated with dronedarone. They did not take into account the fact that there is no data that amiodarone, the most potent antiarrhythmic agent on the market, has no data for reduction in hospitalizations, and, in fact, has data to the contrary. ${ }^{61}$ Dronedarone was also turned down by the British National Institute for Health and Clinical Excellence for similar reasons.

\section{Conclusions}

AF is increasingly prevalent in our society. It brings significant morbidity and increases mortality. Treatment of $\mathrm{AF}$ and its sequelae is costly and needs to be contained. Strategies leading to a lower risk of embolic complications of AF and reduced AF-related hospitalizations need to be developed to meet these goals. While new medications addressing the embolic risks of AF are being introduced on the market, dronedarone, a novel antiarrhythmic agent, has been shown to both reduce the risk of stroke and the rate of hospital presentations and admissions among the patients suffering from AF. Although its use may be more limited than initially expected, it will likely reduce the cost of care for appropriately selected AF patients. Future after-market studies similar to that reported by the Humana registry would shed further light on whether dronedarone can indeed lower the cost of AF care in our society. 


\section{Disclosure}

The authors report no conflicts of interest in this work.

\section{References}

1. Camm AJ, Obel OA. Epidemiology and mechanism of atrial fibrillation and atrial flutter. Am J Cardiol. 1996;78(8A):3-11.

2. Naccarelli GV, Varker H, Lin J, Schulman KL. Increasing prevalence of atrial fibrillation and flutter in the United States. Am J Cardiol. 2009;104(11):1534-1539.

3. Go AS, Hylek EM, Phillips KA, et al. Prevalence of diagnosed atrial fibrillation in adults: national implications for rhythm management and stroke prevention: the AnTicoagulation and Risk Factors in Atrial Fibrillation (ATRIA) Study. JAMA. 2001;285(18) 2370-2375.

4. Bialy D, Lehmann MH, Schumacher DN, Steinman RT, Meissner MD. Hospitalization for arrhythmias in the United States: importance of atrial fibrillation. J Am Coll Cardiol. 1992;19(3):41A.

5. Olivotto I, Cecchi F, Casey SA, Dolara A, Traverse JH, Maron BJ. Impact of atrial fibrillation on the clinical course of hypertrophic cardiomyopathy. Circulation. 2001;104(21):2517-2524.

6. Wolf PA, Mitchell JB, Baker CS, Kannel WB, D'Agostino RB. Impact of atrial fibrillation on mortality, stroke, and medical costs. Arch Intern Med. 1998;158(3):229-234.

7. Olesen JB, Lip GY, Hansen ML, et al. Validation of risk stratification schemes for predicting stroke and thromboembolism in patients with atrial fibrillation: nationwide cohort study. BMJ. 2011;342:d124.

8. Benjamin EJ, Wolf PA, D'Agostino RB, Silbershatz H, Kannel WB, Levy D. Impact of atrial fibrillation on the risk of death: the Framingham Heart Study. Circulation. 1998;98(10):946-952.

9. Ringborg A, Nieuwlaat R, Lindgren P, et al. Costs of atrial fibrillation in five European countries: results from the Euro Heart Survey on atrial fibrillation. Europace. 2008;10(4):403-411.

10. Chung MK, Shemanski L, Sherman DG, et al. Functional status in rate- versus rhythm-control strategies for atrial fibrillation: results of the Atrial Fibrillation Follow-Up Investigation of Rhythm Management (AFFIRM) Functional Status Substudy. J Am Coll Cardiol. 2005;46(10):1891-1899.

11. Corley SD, Epstein AE, DiMarco JP, et al. Relationships between sinus rhythm, treatment, and survival in the Atrial Fibrillation Follow-Up Investigation of Rhythm Management (AFFIRM) Study. Circulation. 2004;109(12):1509-1513.

12. Guglin M, Chen R, Curtis AB. Sinus rhythm is associated with fewer heart failure symptoms: insights from the AFFIRM trial. Heart Rhythm. 2010;7(5):596-601.

13. Roy D, Talajic M, Dorian P, et al. Amiodarone to prevent recurrence of atrial fibrillation. Canadian Trial of Atrial Fibrillation Investigators. N Engl J Med. 2000;342(13):913-920.

14. Vassallo P, Trohman RG. Prescribing amiodarone: an evidence-based review of clinical indications. JAMA. 2007;298(11):1312-1322.

15. Nattel S, Singh BN. Evolution, mechanisms, and classification of antiarrhythmic drugs: focus on class III actions. Am J Cardiol. 1999;84(9A):11R-19R.

16. Wilber DJ, Pappone C, Neuzil P, et al. Comparison of antiarrhythmic drug therapy and radiofrequency catheter ablation in patients with paroxysmal atrial fibrillation: a randomized controlled trial. JAMA. 2010;303(4):333-340.

17. Cappato R, Calkins H, Chen SA, et al. Updated worldwide survey on the methods, efficacy, and safety of catheter ablation for human atrial fibrillation. Circ Arrhythm Electrophysiol. 2010;3(1):32-38.

18. Van Gelder IC, Groenveld HF, Crijns HJ, et al. Lenient versus strict rate control in patients with atrial fibrillation. $N$ Engl J Med. 2010;362(15):1363-1373.

19. Gillis AM, Verma A, Talajic M, Nattel S, Dorian P. Canadian Cardiovascular Society atrial fibrillation guidelines 2010: rate and rhythm management. Can J Cardiol. 2011;27(1):47-59.
20. Stewart S, Murphy NF, Walker A, McGuire A, McMurray JJ. Cost of an emerging epidemic: an economic analysis of atrial fibrillation in the UK. Heart. 2004;90(3):286-292.

21. Hart RG, Pearce LA, Aguilar MI. Meta-analysis: antithrombotic therapy to prevent stroke in patients who have nonvalvular atrial fibrillation. Ann Intern Med. 2007;146(12):857-867.

22. ACTIVE Investigators, Connolly SJ, Pogue J, Hart RG, et al. Effect of clopidogrel added to aspirin in patients with atrial fibrillation. $N$ Engl J Med. 2009;360(20):2066-2078.

23. Connolly SJ, Ezekowitz MD, Yusuf S, et al. Dabigatran versus warfarin in patients with atrial fibrillation. $N$ Engl J Med. 2009;361(12): 1139-1151.

24. Blackshear JL, Odell JA. Appendage obliteration to reduce stroke in cardiac surgical patients with atrial fibrillation. Ann Thorac Surg. 1996;61(2):755-759.

25. Reddy VY, Holmes D, Doshi SK, Neuzil P, Kar S. Safety of percutaneous left atrial appendage closure: results from the Watchman Left Atrial Appendage System for Embolic Protection in Patients with AF (PROTECT AF) clinical trial and the Continued Access Registry. Circulation. 2011;123(4):417-424.

26. Coyne KS, Paramore C, Grandy S, Mercader M, Reynolds M, Zimetbaum P. Assessing the direct costs of treating nonvalvular atrial fibrillation in the United States. Value Health. 2006;9(5):348-356.

27. Bruggenjurgen B, Rossnagel K, Roll S, et al. The impact of atrial fibrillation on the cost of stroke: the berlin acute stroke study. Value Health. 2007;10(2):137-143.

28. Canadian Institute for Health Information. The Cost of Acute Care Hospital Stays by Medical Condition in Canada, 2004-2005. Available from: http://secure.cihi.ca/cihiweb/products/nhex_acutecare07_e.pdf. Accessed December 29, 2011.

29. Wu EQ, Birnbaum HG, Mareva M, et al. Economic burden and comorbidities of atrial fibrillation in a privately insured population. Curr Med Res Opin. 2005;21(10):1693-1699.

30. Naccarelli GV, Johnston SS, Lin J, Patel PP, Schulman KL. Cost burden of cardiovascular hospitalization and mortality in ATHENA-like patients with atrial fibrillation/atrial flutter in the United States. Clin Cardiol. 2010;33(5):270-279.

31. Reinhold T, Lindig C, Willich SN, Bruggenjurgen B. The costs of atrial fibrillation in patients with cardiovascular comorbidities - a longitudinal analysis of German health insurance data. Europace. 2011;13(9):1275-1280.

32. Holstenson E, Ringborg A, Lindgren P, et al. Predictors of costs related to cardiovascular disease among patients with atrial fibrillation in five European countries. Europace. 2011;13(1):23-30.

33. Khaykin Y, Morillo CA, Skanes AC, McCracken A, Humphries K, Kerr CR. Cost comparison of catheter ablation and medical therapy in atrial fibrillation. J Cardiovasc Electrophysiol. 2007;18(9):907-913.

34. Moeremans K, Aliott E, Chillou Cd, Annemans L, Pen CL, Jong Pd. Second line pharmacological management of paroxysmal and persistent atrial fibrillation in france: a cost analysis. Value Health. 2000;3(6): 407-416.

35. Gladstone DJ, Bui E, Fang J, et al. Potentially preventable strokes in high-risk patients with atrial fibrillation who are not adequately anticoagulated. Stroke. 2009;40(1):235-240.

36. Granger CB, Alexander JH, McMurray JJ, et al. Apixaban versus warfarin in patients with atrial fibrillation. $N$ Engl J Med. 2011;365(11): 981-992.

37. Sorensen SV, Kansal AR, Connolly S, et al. Cost-effectiveness of dabigatran etexilate for the prevention of stroke and systemic embolism in atrial fibrillation: a Canadian payer perspective. Thromb Haemost. 2011;105(5):908-919.

38. Pink J, Lane S, Pirmohamed M, Hughes DA. Dabigatran etexilate versus warfarin in management of non-valvular atrial fibrillation in UK context: quantitative benefit-harm and economic analyses. $B M J$. 2011;343:d6333.

39. Shah SV, Gage BF. Cost-effectiveness of dabigatran for stroke prophylaxis in atrial fibrillation. Circulation. 2011;123(22):2562-2570. 
40. Haissaguerre M, Jais P, Shah DC, et al. Spontaneous initiation of atrial fibrillation by ectopic beats originating in the pulmonary veins. $N \mathrm{Engl}$ J Med. 1998;339(10):659-666.

41. Nademanee K, McKenzie J, Kosar E, et al. A new approach for catheter ablation of atrial fibrillation: mapping of the electrophysiologic substrate. J Am Coll Cardiol. 2004;43(11):2044-2053.

42. Nakagawa H, Scherlag BJ, Patterson E, Ikeda A, Lockwood D, Jackman WM. Pathophysiologic basis of autonomic ganglionated plexus ablation in patients with atrial fibrillation. Heart Rhythm. 2009; 6(12 Suppl):S26-S34.

43. Wazni OM, Marrouche NF, Martin DO, et al. Radiofrequency ablation vs antiarrhythmic drugs as first-line treatment of symptomatic atrial fibrillation: a randomized trial. JAMA. 2005;293(21):2634-2640.

44. Jais P, Cauchemez B, Macle L, et al. Catheter ablation versus antiarrhythmic drugs for atrial fibrillation: the A4 study. Circulation. 2008;118(24):2498-2505.

45. Reynolds MR, Essebag V, Zimetbaum P, Cohen DJ. Healthcare resource utilization and costs associated with recurrent episodes of atrial fibrillation: the FRACTAL registry. J Cardiovasc Electrophysiol. 2007;18(6):628-633.

46. Chan PS, Vijan S, Morady F, Oral H. Cost-effectiveness of radiofrequency catheter ablation for atrial fibrillation. J Am Coll Cardiol. 2006;47(12):2513-2520.

47. Eckard N, Davidson T, Walfridsson H, Levin AL. Cost-effectiveness of catheter ablation treatment for patients with symptomatic atrial fibrillation. J Atr Fibrillation. 2009;1(8):461-470.

48. McKenna C, Palmer S, Rodgers M, et al. Cost-effectiveness of radiofrequency catheter ablation for the treatment of atrial fibrillation in the United Kingdom. Heart. 2009;95(7):542-549.

49. Reynolds MR, Zimetbaum P, Josephson ME, Ellis E, Danilov T, Cohen DJ. Cost-effectiveness of radiofrequency catheter ablation compared with antiarrhythmic drug therapy for paroxysmal atrial fibrillation. Circ Arrhythm Electrophysiol. 2009;2(4):362-369.

50. Kathofer S, Thomas D, Karle CA. The novel antiarrhythmic drug dronedarone: comparison with amiodarone. Cardiovasc Drug Rev. 2005;23(3):217-230.

51. Davy JM, Herold M, Hoglund C, et al. Dronedarone for the control of ventricular rate in permanent atrial fibrillation: the Efficacy and safety of dRonedArone for the cOntrol of ventricular rate during atrial fibrillation (ERATO) study. Am Heart J. 2008;156(3):527. e521-e529.

52. Hohnloser SH, Crijns HJ, van Eickels M, et al. Effect of dronedarone on cardiovascular events in atrial fibrillation. $N$ Engl J Med. 2009;360(7):668-678.

53. Torp-Pedersen C, Crijns HJ, Gaudin C, Page RL, Connolly SJ, Hohnloser SH; ATHENA Investigators. Impact of dronedarone on hospitalization burden in patients with atrial fibrillation: results from the ATHENA study. Europace. 2011;13(8):1118-1126.
54. NHS National Institute for Health and Clinical Excellence. Dronedarone for the treatment of nonpermanent atrial fibrillation (TA197). 2010. Available from: http://publications.nice.org.uk/dronedarone-for-thetreatment-of-nonpermanent-atrial-fibrillation-ta197/guidance. Accessed December 10, 2011.

55. Kober L, Torp-Pedersen C, McMurray JJ, et al. Increased mortality after dronedarone therapy for severe heart failure. $N$ Engl J Med. 2008;358(25):2678-2687.

56. FDA. FDA Drug Safety Communication: Multaq (dronedarone) and increased risk of death and serious cardiovascular adverse events. 2011. Available from: http://www.fda.gov/Drugs/DrugSafety/ucm264059. htm. Accessed November 2, 2011.

57. FDA. FDA Drug Safety Communication: Severe liver injury associated with the use of dronedarone (marketed as Multaq). 2011. Available from: http://www.fda.gov/drugs/drugsafety/ucm240011.htm. Accessed November 2, 2011.

58. Piccini JP, Hasselblad V, Peterson ED, Washam JB, Califf RM, Kong DF. Comparative efficacy of dronedarone and amiodarone for the maintenance of sinus rhythm in patients with atrial fibrillation. J Am Coll Cardiol. 2009;54(12):1089-1095.

59. Le Heuzey JY, De Ferrari GM, Radzik D, Santini M, Zhu J, Davy JM. A short-term, randomized, double-blind, parallel-group study to evaluate the efficacy and safety of dronedarone versus amiodarone in patients with persistent atrial fibrillation: the DIONYSOS study. J Cardiovasc Electrophysiol. 2010;21(6):597-605.

60. Canadian Agency for Drugs and Technologies in Health. CEDAC FINAL RECOMMENDATION on DRONEDARONE HYDROCHLORIDE. Common Drug Review. 2010. Available from: http://www. cadth.ca/media/cdr/complete/cdr_complete_Multaq_May-31-2010. pdf. Accessed December 10, 2011.

61. Saksena S, Slee A, Waldo AL, et al. Cardiovascular outcomes in the AFFIRM Trial (Atrial Fibrillation Follow-Up Investigation of Rhythm Management). An assessment of individual antiarrhythmic drug therapies compared with rate control with propensity score-matched analyses. J Am Coll Cardiol. 2011;58(19):1975-1985.

62. Touboul P, Brugada J, Capucci A, Crijns HJ, Edvardsson N, Hohnloser SH. Dronedarone for prevention of atrial fibrillation: a dose-ranging study. Eur Heart J. 2003;24(16):1481-1487.

63. Kober L, Torp-Pedersen C, McMurray JJ, et al. Increased mortality after dronedarone therapy for severe heart failure. $N$ Engl J Med. 2008;358(25):2678-2687.

64. Singh BN, Connolly SJ, Crijns HJ, et al. Dronedarone for maintenance of sinus rhythm in atrial fibrillation or flutter. $N$ Engl J Med. 2007;357(10):987-999.
ClinicoEconomics and Outcomes Research

\section{Publish your work in this journal}

ClinicoEconomics \& Outcomes Research is an international, peerreviewed open-access journal focusing on Health Technology Assessment, Pharmacoeconomics and Outcomes Research in the areas of diagnosis, medical devices, and clinical, surgical and pharmacological intervention. The economic impact of health policy and health systems

\section{Dovepress}

organization also constitute important areas of coverage. The manuscript management system is completely online and includes a very quick and fair peer-review system, which is all easy to use. Visit http://www.dovepress.com/testimonials.php to read real quotes from published authors. 